\title{
Recent Searches for Beyond Standard Model Effects at BABAR
}

\author{
Gerald Eigen*t \\ Department of Physics, University of Bergen, 5007 Bergen, Norway \\ E-mail: gerald.eigen@ift.uib.no \\ On behalf of the BABAR Collaboration
}

In the recent past, several hints of inconsistencies between experimental results and theory predictions in the framework of the Standard Model (SM) have been obtained from measurement of $B$-meson decays, though no beyond-SM (BSM) effects have been observed yet. We report here about a few recent searches for BSM effects in B-meson decays performed with the full BaBar data sample, collected at the energy of the $\Upsilon(4 S)$ resonance, which corresponds to 471 million $B \bar{B}$ pairs. Among these measurements of semileptonic decays involving the tau lepton, which evidenced a significant discrepancy with the SM predictions, and of the rare flavour changing neutral current processes $B \rightarrow K^{(*)} l^{+} l^{-}$where $l=e, \mu, \tau$. The latter, highly suppressed in the SM, occur at lowest order via 1-loop diagrams, and contributions from virtual particles in the loop allow one to probe large mass scales at relatively low energies. In particular, the decays $B \rightarrow K^{*} e^{+} e^{-}$and $B \rightarrow K^{*} \mu^{+} \mu^{-}$(both charged and neutral modes) are studied using an angular analysis to extract the quantities $A_{\mathrm{FB}}$ and $F_{\mathrm{L}}$, and the quantity $P_{2}$, which is subject to smaller theoretical uncertainties and is more sensitive to non-SM contributions. We also present a search for the not yet observed $B^{+} \rightarrow K^{+} \tau^{+} \tau^{-}$decay.

The European Physical Society Conference on High Energy Physics

5-12 July, 2017

Venice

\footnotetext{
* Speaker.

$\dagger$ This work was supported by Norwegian Research Council. I would like to thank the BABAR collaboration for the opportunity to present these results, in particular I would like to thank J. McKenna , J. Albert F.C. Porter for useful discussions.
} 


\section{Introduction}

The flavor-changing neutral current (FCNC) decays $B \rightarrow K^{(*)} \ell^{+} \ell^{-}$where $\ell^{ \pm}=e^{ \pm}, \mu^{ \pm}, \tau^{ \pm}$ are forbidden in the SM at tree level. They proceed at higher orders via penguin loops and box diagrams. New physics (NP) effects introduce new loops with new particles. These decays probe new physics effects at a scale of a few TeV. In particular, angular observables bear high sensitivity to NP contributions. The decay $B^{ \pm} \rightarrow K^{ \pm} \tau^{+} \tau^{-}$is highly suppressed in the SM and the $\tau$ may couple more strongly to NP processes than to light leptons. In both analyses we use the full BABAR data sample of $(471 \pm 3) \times 10^{6} B \bar{B}$ events.

\section{Angular Asymmetries in the Decays $B \rightarrow K^{*} \ell^{+} \ell^{-}$}

The $B \rightarrow K^{*} \ell^{+} \ell^{-}$angular distribution depends on three angles: $\theta_{K}$, the angle between the Kaon and the $B$ meson in $K^{*}$ rest frame; $\theta_{\ell}$, the angle between $\ell^{+}\left(\ell^{-}\right)$and the $B(\bar{B})$ meson in $\ell^{+} \ell^{-}$rest frame; $\phi$, the angle between the di-lepton and the $K \pi$ decay planes. The full angular distribution involves $12 q^{2}$-dependent parameters [1]. Since the BABAR data sample is too small to measure them all in different regions of $q^{2}$, we fit the one-dimensional projections

$$
\begin{aligned}
W\left(\cos \theta_{K}\right) & =\frac{3}{2} F_{\mathrm{L}}\left(q^{2}\right) \cos ^{2} \theta_{K}+\frac{3}{4}\left(1-F_{\mathrm{L}}\left(q^{2}\right)\right) \sin ^{2} \theta_{K} \\
W\left(\cos \theta_{\ell}\right) & =\frac{3}{4} F_{\mathrm{L}}\left(q^{2}\right) \sin ^{2} \theta_{\ell}+\frac{3}{8}\left(1-F_{\mathrm{L}}\left(q^{2}\right)\right)\left(1+\cos ^{2} \theta_{\ell}\right)+A_{\mathrm{FB}}\left(q^{2}\right) \cos \theta_{\ell},
\end{aligned}
$$

which depend only on the $K^{*}$ longitudinal polarization, $F_{\mathrm{L}}\left(q^{2}\right)$, and the lepton forward-backward asymmetry, $A_{\mathrm{FB}}\left(q^{2}\right)$. We measure these in five disjoint $q^{2}$ bins and in $q_{0}^{2}=1-6 \mathrm{GeV}^{2}$. We further determine $P_{2}\left(q^{2}\right)=(-2 / 3) \times A_{\mathrm{FB}}\left(q^{2}\right) /\left(1-F_{\mathrm{L}}\left(q^{2}\right)\right)$ that has smaller theory errors.

We reconstruct five final states: $B^{+} \rightarrow K^{*+}\left(\rightarrow K_{S}^{0} \pi^{+}\right) \mu^{+} \mu^{-}, B^{0} \rightarrow K^{* 0}\left(\rightarrow K^{+} \pi^{-}\right) \mu^{+} \mu^{-}$, $B^{+} \rightarrow K^{*+}\left(\rightarrow K_{S}^{0} \pi^{+}\right) e^{+} e^{-}, B^{+} \rightarrow K^{*+}\left(\rightarrow K^{+} \pi^{0}\right) e^{+} e^{-}$and $B^{0} \rightarrow K^{* 0}\left(\rightarrow K^{+} \pi^{-}\right) e^{+} e^{-}$[2]. We employ standard requirements on $m_{\mathrm{ES}}$ (beam-energy constrained mass) and $\Delta E$ (energy difference between $B$ meson and beam energies) to select signal. To reduce $B \bar{B}$ and $e^{+} e^{-} \rightarrow q \bar{q}$ background contributions we define eight bagged decision trees with ten input variables for $e^{+} e^{-}$and $\mu^{+} \mu^{-}$ modes for low and high $q^{2}$. We combine the $B \bar{B}$ BDT outputs into a likelihood ratio $L_{\mathrm{R}}$. First, we perform an initial three-dimensional maximum-likelihood fit to $m_{\mathrm{ES}}, m_{K \pi}$ and $L_{\mathrm{R}}$ for each mode separately. We fix the normalization and probability density functions (pdf) for $m_{\mathrm{ES}}, m_{K \pi}$ and $L_{\mathrm{R}}$. Figure 1 shows the $m_{\mathrm{ES}}, m_{K \pi}$ and $L_{\mathrm{R}}$ projections for $B^{0} \rightarrow K^{* 0}\left(\rightarrow K^{+} \pi^{-}\right) e^{+} e^{-}$in the last bin $q^{2}$ with results of the initial fit overlaid. We repeat these fits in the angular-fit signal region $\left(m_{\mathrm{ES}}>\right.$ $\left.5.27 \mathrm{GeV} / \mathrm{c}^{2}\right)$. We then add $\cos \theta_{K}$ as a fourth variable to the likelihood function with $F_{\mathrm{L}}\left(q^{2}\right)$ as the only free parameter keeping all other parameters fixed. In the last step we replace $\cos \theta_{K}$ with $\cos \theta_{\ell}$ as the fourth variable in the likelihood function with $A_{\mathrm{FB}}$ as the only free parameter keeping all other parameters and $F_{\mathrm{L}}\left(q^{2}\right)$ fixed. We determine each angular result subsequently by direct construction and examination of the $\log (L)$ curves from a scan across entire $F_{\mathrm{L}}\left(q^{2}\right)$ and $A_{\mathrm{FB}}\left(q^{2}\right)$ parameter space. We characterize the pdfs for $m_{\mathrm{ES}}, m(K \pi), L_{\mathrm{R}}, \cos \theta_{K}$ and $\cos \theta_{\ell}$ for correctly reconstructed signal events, for random combinatorial backgrounds, for backgrounds from $J / \psi K^{*}$ and $\psi(2 S) K^{*}$ decays and for backgrounds in which hadrons are misidentified as muons. 

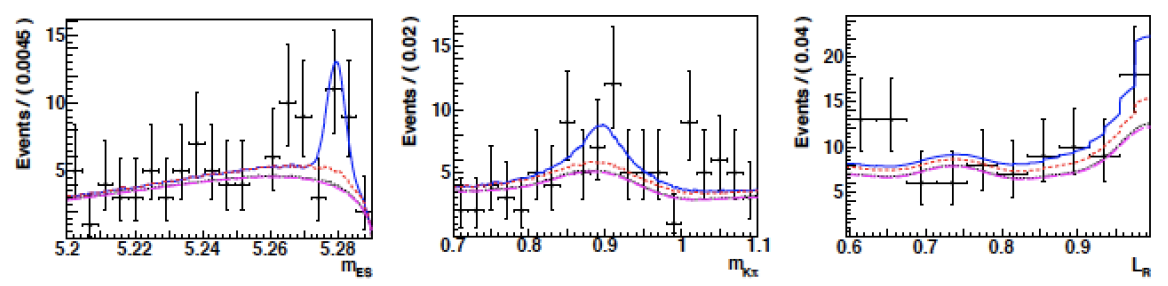

Figure 1: Distributions of $m_{\mathrm{ES}}$ (left), $m_{K \pi}$ (middle) and $L_{\mathrm{R}}$ (right) for data (points), total fit (blue line), combinatorial background (magenta dashes), charmonium feed-down (black dots) and cross-feed (red dashes).

Figure 2 shows our results for $F_{\mathrm{L}}\left(q^{2}\right), A_{\mathrm{FB}}(q 2)$ and $P_{2}\left(q^{2}\right)$ for $B^{+}$modes, $B^{0}$ modes and their combination [2] for five bins of $q^{2}$ in comparison to the SM prediction [3] and measurements from Belle [4], CDF [5], CMS [6], ATLAS [7] and LHCb [8, 9]. For $B^{+}$modes $F_{\mathrm{L}}\left(q^{2}\right)$ below the $J / \psi$ shows deviations from the SM predictions that are $>3 \sigma$ for the second $q^{2}$ bin while $A_{\mathrm{FB}}\left(q^{2}\right)$ and $P_{2}\left(q^{2}\right)$ are consistent with the SM. For $B^{0}$ modes $F_{\mathrm{L}}\left(q^{2}\right)$ is consistent with the SM except for the second $q^{2}$ bin where the discrepancy is $>3 \sigma$ whereas $A_{\mathrm{FB}}\left(q^{2}\right)$ and $P_{2}\left(q^{2}\right)$ agree with the SM prediction. The latest LHCb measurements of $F_{\mathrm{L}}\left(q^{2}\right)$ and $A_{\mathrm{FB}}\left(q^{2}\right)$ measured in the decay $B^{0} \rightarrow K^{* 0} \mu^{+} \mu^{-}$show good agreement with the SM prediction.
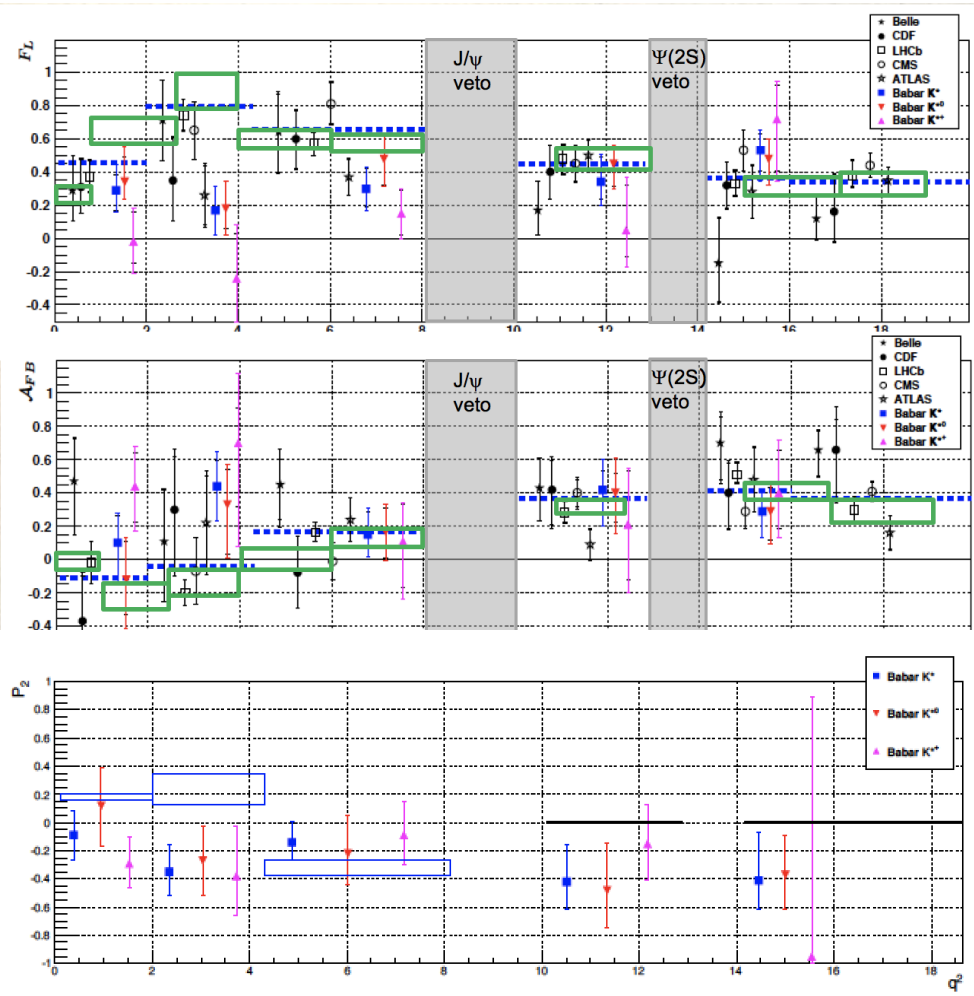

Figure 2: $B A B A R$ measurements of $F_{\mathrm{L}}\left(q^{2}\right)$ (top), $A_{\mathrm{FB}}\left(q^{2}\right)$ (middle) and $P_{2}\left(q^{2}\right)$ (bottom) for $B^{+}$modes (magenta upward triangle) , $B^{0}$ modes (red downward triangle) and their combination (blue square) in comparison to the SM prediction (blue dash/blue box) defining also the extent of the $q^{2}$ bin, and results from Belle (black star), CDF (black point), LHCb (open square/green box), CMS (open circle) and ATLAS (large star). The grey-shaded regions show the $J / \psi$ and $\psi(2 S)$ vetoes. 


\section{Search for the Decay $B^{ \pm} \rightarrow K^{ \pm} \tau^{+} \tau^{-}$}

We tag one $B$ meson via full hadronic reconstruction $\left(B_{\mathrm{tag}}\right)$ using standard $m_{\mathrm{ES}}$ and $\Delta E$ selections and look for $B^{+} \rightarrow K^{+} \tau^{+} \tau^{-}$signal in the recoil [10]. The $B_{\text {tag }}$ accounts for 2968 hadronic decays of $B \rightarrow X_{c} X$ with $\left[X_{c}=D^{(*) 0}, D^{(*) \pm}, D_{s}^{(*) \pm}, J / \psi ; X \leq 5\left(K^{ \pm, 0}+\pi^{ \pm, 0}\right)\right]$. Reconstructing both taus in purely leptonic modes, we require exactly three charged tracks with the correct particle identification plus missing energy. We remove $75 \%$ of the $q \bar{q}$ background while retaining $80 \%$ of all $B \bar{B}$ events with the help of a multivariate likelihood ratio in which $B \bar{B}$ pdfs and $q \bar{q}$ pdfs for six event shape variables are combined. We remove events for which the $K \mp \ell^{ \pm}$mass is consistent with a $D^{0}$, the di-lepton mass is consistent with a $J / \psi$ or the summed energy in the electromagnetic calorimeter is larger than $50 \mathrm{MeV}$. We reduce the main peaking background from $B \rightarrow \bar{D}^{(*)} \ell^{+} v$ with $\bar{D}^{(*)} \rightarrow K \ell^{\prime} \bar{v}$ with a multi-layer perceptron (MLP) neural network trained with eight kinematic and event shape variables. The dominant systematic uncertainties result from particle ID, the $\pi^{0}$ veto, theory and MLP neural network. The observed yield is $176 \pm 13$ events compared to the expected background of $154.4 \pm 7.1$ events. The excess is at the $2 \sigma$ level and we set a branching fraction upper limit of $\mathscr{B}\left(B^{+} \rightarrow K^{+} \tau^{+} \tau^{-}\right)<2.25 \times 10^{-3} @ 90 \%$ confidence level (CL).

\section{Conclusion}

We measured the angular distributions of $B^{+} \rightarrow K^{*+} \ell^{+} \ell^{-}$and $B^{0} \rightarrow K^{* 0} \ell^{+} \ell^{-}$extracting the $F_{\mathrm{L}}\left(q^{2}\right), A_{\mathrm{FB}}\left(q^{2}\right)$ and $P_{2}\left(q^{2}\right)$ in five disjoint bins of $q^{2}$ and for $q_{0}^{2}=1-6 \mathrm{GeV}^{2}$. For $F_{\mathrm{L}}\left(q^{2}\right)$ we see deviations from the SM for $q^{2}$ below the $J / \psi$, in particular for $B^{+}$modes, whereas $A_{\mathrm{FB}}\left(q^{2}\right)$ and $P_{2}\left(q^{2}\right)$ are consistent with the SM. We searched for $B^{ \pm} \rightarrow K^{ \pm} \tau^{+} \tau^{-}$and set a branching fraction upper limit of $\mathscr{B}\left(B^{+} \rightarrow K^{+} \tau^{+} \tau^{-}\right)<2.25 \times 10^{-3} @ 90 \%$ CL.

\section{References}

[1] J. Matias et al., JHEP1204, 104 (2012) and references therein.

[2] The BABAR Collaboration (J.P. Lees et al., Phys. Rev. D93, 052015 (2016).

[3] C. Bobeth et al., JHEP 1107, 067 (2011); M Beneke et al., Nucl. Phys. B612, 25 (2005); B. Grindstein and D. Pirjol, Phys. Rev. D70, 114005 (2004); S. Descotes-Genon et al., JHEP 1305, 137 (2013); M. Beylich et al., Eur. Phys. J. C71, 1635 (2011); A. Khodjamirian et al., JHEP 1009, 089 (2010); P. Ball and R. Zwicky, Phys. Rev. D71, 014029 (2005).

[4] The Belle Collaboration (J. T. Wei et al.), Phys. Rev. Lett 103, 171801 (2012).

[5] The CDF Collaboration (T. Aaltonen et al.), Phys. Rev. Lett. 108, 081807 (2012).

[6] The CMS Collaboration (S. Chatrchyan et al.), Phys. Lett. B727, 77 (2013).

[7] The ATLAS Collaboration (G. Aad et al.), ATLAS-Conf-2013-038 (2013).

[8] The LHCb Collaboration (R. Aaij et al.), JHEP 1308, 131 (2013).

[9] The LHCb Collaboration (R. Aaij et al.), JHEP 1602, 65 (2016).

[10] The BABAR Collaboration (J.P. Lees et al., Phys. Rev. Lett. 118, 031802 (2017). 